Communications

in Computer and Information Science

129 
Joaquim Filipe Ana Fred Bernadette Sharp (Eds.)

\section{Agents and \\ Artificial Intelligence}

Second International Conference, ICAART 2010 Valencia, Spain, January 22-24, 2010

Revised Selected Papers 
Volume Editors

Joaquim Filipe

INSTICC and IPS

Estefanilha, Setúbal, Portugal

E-mail: joaquim.filipe@estsetubal.ips.pt

Ana Fred

IST - Technical University of Lisbon

Lisbon, Portugal

E-mail: afred@1x.it.pt

Bernadette Sharp

Staffordshire University

Beaconside, Stafford, UK

E-mail: b.sharp@staffs.ac.uk

ISSN 1865-0929

e-ISSN 1865-0937

ISBN 978-3-642-19889-2

DOI 10.1007/978-3-642-19890-8

Springer Heidelberg Dordrecht London New York

Library of Congress Control Number: 2011923680

CR Subject Classification (1998): I.2, I.2.6, H.3-5, C.2, F.1, J.1

C) Springer-Verlag Berlin Heidelberg 2011

This work is subject to copyright. All rights are reserved, whether the whole or part of the material is concerned, specifically the rights of translation, reprinting, re-use of illustrations, recitation, broadcasting, reproduction on microfilms or in any other way, and storage in data banks. Duplication of this publication or parts thereof is permitted only under the provisions of the German Copyright Law of September 9, 1965, in its current version, and permission for use must always be obtained from Springer. Violations are liable to prosecution under the German Copyright Law.

The use of general descriptive names, registered names, trademarks, etc. in this publication does not imply, even in the absence of a specific statement, that such names are exempt from the relevant protective laws and regulations and therefore free for general use.

Typesetting: Camera-ready by author, data conversion by Scientific Publishing Services, Chennai, India

Printed on acid-free paper

Springer is part of Springer Science+Business Media (www.springer.com) 


\section{Preface}

The present book includes a set of selected papers from the Second International Conference on Agents and Artificial Intelligence (ICAART 2010), held in Valencia, Spain, during January 22-24, 2010. The conference was organized in two simultaneous tracks: Artificial Intelligence and Agents. The book is based on the same structure.

ICAART 2010 received 364 paper submissions, from 58 different countries in all continents. From these, after a blind review process, only 31 were accepted as full papers, of which 17 were selected for inclusion in this book, based on the classifications provided by the Program Committee. The selected papers reflect the interdisciplinary nature of the conference. The diversity of topics is an important feature of this conference, enabling an overall perception of several important scientific and technological trends. These high-quality standards will be maintained and reinforced at ICAART 2011, to be held in Rome, Italy, and in future editions of this conference.

Furthermore, ICAART 2010 included five plenary keynote lectures given by Yves Demazeau (Laboratoire d'Informatique de Grenoble), Tim Finin (University of Maryland), Vicent J. Botti i Navarro (Universidad Politécnica de Valencia), Peter D. Karp (Bioinformatics Research Group within the Artificial Intelligence Center at SRI International) and Amilcar Cardoso (University of Coimbra). We would like to express our appreciation to all of them and in particular to those who took the time to contribute with a paper to this book.

On behalf of the conference Organizing Committee, we would like to thank all participants. First of all the authors, whose quality work is the essence of the conference and the members of the Program Committee, who helped us with their expertise and diligence in reviewing the papers. As we all know, producing a conference requires the effort of many individuals. We wish to thank also all the members of our Organizing Committee, whose work and commitment were invaluable.

July 2010

Joaquim Filipe

Ana Fred

Bernadette Sharp 


\section{Conference Committee}

\section{Conference Co-chairs}

Joaquim Filipe

Ana Fred

\section{Program Chair}

Bernadette Sharp

\section{Organizing Committee}

Patrícia Alves

Sérgio Brissos

Helder Coelhas

Andreia Costa

Bruno Encarnação

Bárbara Lima

Liliana Medina

Elton Mendes

Carla Mota

Vitor Pedrosa

José Varela

Pedro Varela

\section{Program Committee}

Stéphane Airiau, The Netherlands Natasha Alechina, UK

Sander Bohte, The Netherlands

Luigi Ceccaroni, Spain

Berndt Farwer, UK

Nicoletta Fornara, Switzerland

Cees Witteveen, The Netherlands

Thomas Ågotnes, Norway

Rudolf Albrecht, Austria

Cesar Analide, Portugal

Andreas Andreou, Cyprus

Ioannis N. Athanasiadis, Switzerland
Polytechnic Institute of Setúbal /

INSTICC, Portugal

Technical University of Lisbon / IT, Portugal

Staffordshire University, UK

INSTICC, Portugal

INSTICC, Portugal

INSTICC, Portugal

INSTICC, Portugal

INSTICC, Portugal

INSTICC, Portugal

INSTICC, Portugal

INSTICC, Portugal

INSTICC, Portugal

INSTICC, Portugal

INSTICC, Portugal

INSTICC, Portugal

Antonio Bahamonde, Spain

Matteo Baldoni, Italy

Bikramjit Banerjee, USA

Ana Lúcia C. Bazzan, Brazil

Orlando Belo, Portugal

Carole Bernon, France

Daniel Berrar, UK

Ateet Bhalla, India

Olivier Boissier, France

Tibor Bosse, The Netherlands

Luis Botelho, Portugal

Danielle Boulanger, France 
Paolo Bresciani, Belgium

Egon L. van den Broek,

The Netherlands

Silvia Calegari, Italy

Valérie Camps, France

Jose Jesus Castro-schez, Spain

Radovan Cervenka, Slovak Republic

Georgios Chalkiadakis, UK

Shyi-Ming Chen, Taiwan

Carlos Iván Chesñevar, Argentina

Adam Cheyer, USA

Paolo Ciancarini, Italy

Diane Cook, USA

Paulo Cortez, Portugal

Jacob W. Crandall, UAE

Yves Demazeau, France

Andreas Dengel, Germany

Gaël Dias, Portugal

Ian Dickinson, UK

Edith Elkind, Singapore

Stefano Ferilli, Italy

Antonio Fernández-caballero, Spain

Edilson Ferneda, Brazil

Dariusz Frejlichowski, Poland

Naoki Fukuta, Japan

Wai-Keung Fung, Canada

Arnulfo Alanis Garza, Mexico

Chiara Ghidini, Italy

Joseph Giampapa, USA

Marie-Pierre Gleizes, France

Dominic Greenwood, Switzerland

Sven Groppe, Germany

Zahia Guessoum, France

Renata Guizzardi, Brazil

Andreas Herzig, France

Wladyslaw Homenda, Poland

Wei-Chiang Hong, Taiwan

Mark Hoogendoorn, The Netherlands

Eyke Hüllermeier, Germany

Carlos Iglesias, Spain

François Jacquenet, France

Yanguo Jing, UK

W. Lewis Johnson, USA

Catholijn Jonker, The Netherlands

Lalana Kagal, USA
Matthias Klusch, Germany

Joanna Kolodziej, Poland

Sébastien Konieczny, France

Igor Kotenko, Russian Federation

Stan Kurkovsky, USA

Nuno Lau, Portugal

Ho-fung Leung, Hong Kong

Baoding Liu, China

Chao-Lin Liu, Taiwan

Stephane Loiseau, France

Gabriel Pereira Lopes, Portugal

Hongen Lu, Australia

Bernd Ludwig, Germany

José Machado, Portugal

Nadia Magnenat-thalmann,

Switzerland

Pierre Maret, France

Tokuro Matsuo, Japan

Nicola Di Mauro, Italy

Amnon Meisels, Israel

Benito Mendoza, USA

Daniel Merkle, Denmark

Bernd Meyer, Australia

Simon Miles, UK

Pavlos Moraitis, France

Thierry Moyaux, France

Tracy Mullen, USA

Radhakrishnan Nagarajan, USA

Tomoharu Nakashima, Japan

Paulo Novais, Portugal

Charlie L. Ortiz, USA

Jeng-Shyang Pan, Taiwan

Oscar Pastor, Spain

Krzysztof Patan, Poland

Catherine Pelachaud, France

Wojciech Penczek, Poland

Dana Petcu, Romania

Steve Phelps, UK

Rui Prada, Portugal

Rong Qu, UK

T. Ramayah, Malaysia

Martin Rehák, Czech Republic

Luís Paulo Reis, Portugal

Daniel Rodriguez, Spain

Pilar Rodriguez, Spain 
Rosaldo Rossetti, Portugal

Alessandro Saffiotti, Sweden

Manuel Filipe Santos, Portugal

Jurek Sasiadek, Canada

Camilla Schwind, France

Candace L. Sidner, USA

Peer Olaf Siebers, UK

Viviane Silva, Brazil

Adam Slowik, Poland

Alexander Smirnov,

Russian Federation

Safeeullah Soomro, Saudi Arabia

Kostas Stathis, UK

Kathleen Steinhofel, UK

\section{Auxiliary Reviewers}

Juan José Del Coz, Spain

Jorge Diez, Spain

Alessandro Farinelli, Italy

Ya Gao, China

Sergio Alejandro Gómez, Argentina

Tal Grinshpoun, Israel

Rongqing Huang, China

You Ji, China

Carson K. Leung, Canada

Jinbo Li, China

Oscar Luaces, Spain

\section{Invited Speakers}

Yves Demazeau

Tim Finin

Vicent J. Botti i Navarro

Peter D. Karp

Amilcar Cardoso
Thomas Stützle, Belgium

Chun-Yi Su, Canada

Shiliang Sun, China

Ryszard Tadeusiewicz, Poland

Paola Turci, Italy

Franco Turini, Italy

Paulo Urbano, Portugal

Ioannis Vlahavas, Greece

Wutnipong Warakraisawad, Thailand

Bozena Wozna-Szczesniak, Poland

Seiji Yamada, Japan

Xin-She Yang, UK

Huiyu Zhou, UK
Pedro Moreira, Portugal

Maxime Morge, France

Matthijs Pontier, The Netherlands

José Ramón Quevedo, Spain

Daniel Castro Silva, Portugal

Frans Van der Sluis, The Netherlands

Jung-woo Sohn, USA

Wenting Tu, China

Sylvain Videau, France

Perukrishnen Vytelingum, UK

Zhijie $\mathrm{Xu}$, China
Laboratoire d'Informatique de Grenoble, France

University of Maryland, USA

Universidad Politécnica de Valencia, Spain

Bioinformatics Research Group, Artificial Intelligence Center, SRI International, USA

University of Coimbra, Portugal 


\section{Table of Contents}

\section{Invited Paper}

Creating and Exploiting a Hybrid Knowledge Base for Linked Data ....

Zareen Syed and Tim Finin

\section{Part I: Artificial Intelligence}

Language Support to XML Data Mining: A Case Study . . . . . . . . . . . Andrea Romei and Franco Turini

Improved Door Detection Fusing Camera and Laser Rangefinder Data with AdaBoosting ..........................

Jens Hensler, Michael Blaich, and Oliver Bittel

Combining Color and Spatial Color Distribution Information in a Fuzzy

Rule Based Compact Composite Descriptor .................

Savvas A. Chatzichristofis, Yiannis S. Boutalis, and Mathias Lux

Towards Min Max Generalization in Reinforcement Learning

Raphael Fonteneau, Susan A. Murphy, Louis Wehenkel, and

Damien Ernst

Designing an Epigenetic Approach in Artificial Life: The EpiAL

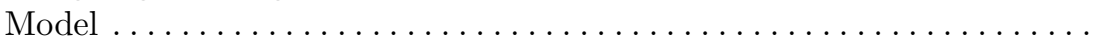

Jorge A.B. Sousa and Ernesto Costa

A Spontaneous Topic Change of Dialogue for Conversational Agent

Based on Human Cognition and Memory .................

Sungsoo Lim, Keunhyun Oh, and Sung-Bae Cho

Clustering Data with Temporal Evolution: Application to

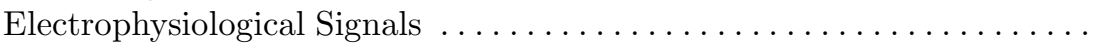

Liliana A.S. Medina and Ana L.N. Fred

\section{Part II: Agents}

MASITS Methodology Supported Development of Agent Based

Intelligent Tutoring System MIPITS . . . . . . . . . . . . . .

Egons Lavendelis and Janis Grundspenkis 
A 3D Indoor Pedestrian Simulator Using an Enhanced Floor Field Model

Chulmin Jun and Hyeyoung Kim

Toward a Self-adaptive Multi-Agent System to Control Dynamic Processes ....................................

Sylvain Videau, Carole Bernon, and Pierre Glize

The Provider Rating Agreement Pattern for Solving the Provider

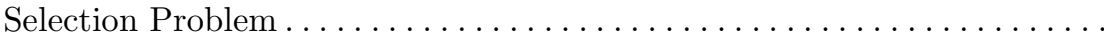

José Javier Durán and Carlos A. Iglesias

Advanced Scheduling Techniques with the Pliant System for High-Level

Grid Brokering ............................

József Dániel Dombi and Attila Kertész

Predictive Learning from Demonstration

Erik A. Billing, Thomas Hellström, and Lars-Erik Janlert

ISReal: A Platform for Intelligent Simulated Realities..............

Stefan Nesbigall, Stefan Warwas, Patrick Kapahnke, René Schubotz,

Matthias Klusch, Klaus Fischer, and Philipp Slusallek

Auction Design and Performance: An Agent-Based Simulation with Endogenous Participation .........................

Atakelty Hailu, John Rolfe, Jill Windle, and Romy Greiner

A Framework for the Development and Maintenance of Adaptive, Dynamic, Context-Aware Information Services . . . . . . . . . . . . . . . . .

Manel Palau, Ignasi Gómez-Sebastià, Luigi Ceccaroni, Javier Vázquez-Salceda, and Juan Carlos Nieves

Decision Making in Complex Systems with an Interdisciplinary

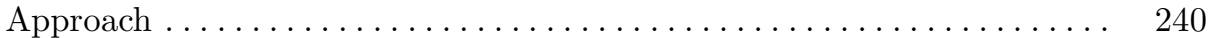

Marina V. Sokolova, Antonio Fernández-Caballero, and Francisco J. Gómez 